\title{
Bounded growth of the bullwhip effect under a class of nonlinear ordering policies
}

\author{
Zhaodong Wang ${ }^{1}$, Xin Wang ${ }^{1}$, and Yanfeng Ouyang*1 \\ ${ }^{1}$ Department of Civil and Environmental Engineering, University of Illinois at Urbana-Champaign, 1209 Newmark \\ Civil Engineering Lab., 205 N. Mathews Ave., Urbana, IL 61801, United States
}

\begin{abstract}
This paper analyzes the bullwhip effect in multi-echelon supply chains under a general class of nonlinear ordering policies. A describing-function approach from control theory is used to derive closed-form formulas to predict amplification of order fluctuations along the supply chain. It is proven that with consideration of nonlinearity in the ordering policy, the magnitude of the bullwhip effect will eventually become bounded after growing through the first few stages of the supply chain. It is also proven that the average customer demand as well as the demand fluctuation frequency would directly affect the bounded magnitude, while the suppliers' demand forecasting method has no effect at all. For illustration, analytical results for a class of order-up-to policies are derived and verified by numerical simulations. The proposed modeling framework holds the promise to not only explain empirical observations, but also serve as the basis for developing counteracting strategies against the bullwhip effect.
\end{abstract}

\section{Introduction}

The bullwhip effect is a well-known phenomenon in supply chains where the amplitude of order fluctuations increases along the chain from downstream to upstream suppliers. In other words, small perturbations in a stable customer demand may cause huge fluctuations in order sequences of the upstream suppliers. First discovered by Forrester (1958), the bullwhip effect has been observed in several areas such as industry operations (Baganha and Cohen, 1998), macroeconomics (Blinder, 1986), and business simulations (Goodwin and Franklin, 1994). A main negative consequence of the bullwhip effect is the significant increase of supply chain operation costs, since the supplier needs to adjust capacity and production plan to meet highly fluctuating orders that he/she receives (Lee et al., 1997; Chen et al., 2000b; Lee et al., 2004; Ouyang and Daganzo, 2006a). Extensive studies have been conducted to explore the mechanism of the bullwhip effect. Most of the literature models order sequences in the time domain as stochastic processes and analyze their statistical properties. Managerial insights on the bullwhip effect are drawn by relating the amplification of order sequence variations (e.g., variance) to various operational causes (Lee et al., 1997; Baganha and Cohen, 1998; Graves, 1999; Chen et al., 2000a,b; Lee et al., 2004; Zhang, 2004; Gaur et al., 2005; Gilbert, 2005). The bullwhip effect is quantified first for simple two-stage supply chain (Chen et al., 2000b) and then extended

${ }^{*}$ Corresponding author. Mail address: 1209 Newmark Civil Engineering Lab., MC-250, 205 N. Mathews Ave, Urbana, IL 61801. Tel.:217-333-9858, Fax: 217-333-1924, Email: yfouyang@illinois.edu 
for multi-echelon supply chain based on Autoregressive Integrated Moving Average (ARIMA) time-series models (Gilbert, 2005).

When investigating the bullwhip effect in practices, people may face the challenge of lacking accurate customer demand information. To this end, frequency domain based robust supply chain analysis is introduced to quantitatively estimate the bullwhip effect as a function of inventory management policies, regardless of the customer demand. A group of literature establishes the theoretical foundation of robust analysis on the bullwhip effect. Daganzo $(2003,2004)$ presents robust analytical results for multi-echelon supply chain systems with general demand inputs. To identify and maintain the system stability, Ouyang (2007) develops robust stability metric for analyzing supply chains with information sharing. To provide quantitative methods to predict the propagation of order fluctuations, variance formulas under linear time-invariant inventory management policies are proposed. For example, Ouyang and Daganzo (2006a, 2008) provide an exact formula to characterize the bullwhip effect for multi-stage supply chains with ergodic customer demand. Later, Ouyang and Li (2010) generalize the formula into a supply chain network including multiple customers, with or without sharing information among suppliers. Also, Dejonckheere et al. $(2003,2004)$ proposes demand-dependent variance formulas for the situation where customer demand are forecasted. Based on the theoretical achievement, Li et al. (2013) propose a dampened trend forecasting method and study the stability under the order-up-to policy. Furthermore, Ivanov and Sokolov (2013) and Fu et al. (2014) conduct dynamic control analysis to avoid the bullwhip effect.

Although the aforementioned studies provide valuable insights, their results highly depend on pre-assumed customer demand processes and linearized ordering policies, which limit their applicability to general supply chains. Unfortunately, in reality, most known optimal ordering policies are actually nonlinear. For example, the $(S, s)$ and order-up-to policies are respectively proven to be optimal in certain supply chain contexts and widely used in practical operations (Karlin, 1958; Clark and Scarf, 1960). Under these policies, the optimal ordering quantity follows a piecewise linear function of the inventory position, and negative orders (i.e., returning of items) are not allowed. The linearized models, on the other hand, may allow negative orders, and as a result, lead to unrealistic or even meaningless bullwhip effect estimates. For example, linearized systems control theory generally conclude that the fluctuations of the order sequences under linearized ordering policies would always increase exponentially as we move along the supply chain (Ouyang and Daganzo, 2006a; Ouyang and Li, 2010; Li and Ouyang, 2011). This has been found to contradict with empirical observations, e.g., (Cachon et al., 2007) found that real-world order fluctuations for upstream suppliers tend to stabilize to bounded values.

It is conjectured that nonlinearity in the ordering policies is key to the bounded amplification in the observed bullwhip effect. Nevertheless, analysis of nonlinear dynamic systems imposes significant difficulties. Among a number of mathematical tools for nonlinear systems, describing function is widely implemented in nonlinear control community and of great advantages when the fluctuation is the focus in system analysis (Kochenburger, 1950). The fundamental idea of the describing function method is quasi-linear approximation, i.e., approximating the output of a nonlinear system as sinusoids when the linear component of the system could serve as a low-pass filter (Davis, 1962; Blaquiere, 2012). Describing-function-based method has also been applied in many contexts such as wireless communication (Jalaleddini and Aghdam, 2010), traffic flow oscillation (Li et al., 2010; Li and Ouyang, 2011; Li et al., 2012), and others. It has been found that the propagation of vehicle speed fluctuations in freeway traffic streams is quite similar to the bullwhip effect in supply chains (Edie, 1961; Ouyang et al., 2006) and further evidences show that traffic flow can be unified with supply chain system in terms of stability analysis (Helbing, 2005; Ouyang et al., 2006). We are inspired to develop a describing-function-based approach (DFA) to study the bullwhip effect under nonlinear ordering policies. This not only fills the gaps between theoretical predictions and empirical observations, but 
also advances methodologies that can be used to overcome the difficulties associated with nonlinear systems analysis. To the authors' best knowledge, no research has been conducted to achieve these goals in the context of supply chain management.

This paper applies the describing function technique to analyze the bullwhip effect under nonlinear ordering policies. We propose system adjustment treatments to the nonlinear supply chain so that the describing function technique is suitable for the analysis. Mathematical closed-form results are derived to predict the propagation of order fluctuations, and the formulas are found to precisely reproduce the empirical observation in multi-echelon supply chains. A refinement method is also proposed to improve the accuracy of the prediction in practical applications. We illustrate the proposed mathematical framework with a class of nonlinear order-up-to policies (i.e., order-up-to policies), derive system properties, and verify the accuracy of the analytical predictions with numerical simulations. The proposed analytical modeling framework is found to not only enhance our understanding of the bounded growth of the bullwhip effect, but also provide insights for developing strategies that can mitigate or even avoid the bullwhip effect.

The remainder of this paper is organized as follows. Section 2 introduces the modeling framework for generalized bullwhip effect analysis under general ordering policies. Section 3 presents an application of the framework to a specific case of order-up-to policy, and demonstrates its accuracy using numerical experiments. Finally, Section 4 concludes the paper and discusses future extensions.

\section{Methodology}

\subsection{Model Formulation}

\subsubsection{System Dynamics}

Consider a typical multi-echelon supply chain with $I+1$ suppliers (e.g., retailer, wholesaler, distributor, manufacturer) and one downstream end customer, as shown in Fig. 1. For convenience, the customer is considered as supplier 0 . At discrete time $t=-\infty, \ldots,-1,0,1, \ldots,+\infty$, every supplier $i=0,1, \ldots$ decides their order quantity $u_{i}(t)$ based on their ordering policy from their upstream neighbor $i+1$, and receives the goods after a constant lead time $l_{i}$. Denoting $x_{i}(t)$ as the supplier's inventory position, $y_{i}(t)$ as the in-stock inventory, we have the following conservation law (Ouyang and Daganzo, 2006a):

$$
\begin{aligned}
& x_{i}(t+1)=x_{i}(t)+u_{i}(t)-u_{i-1}(t), \quad i=1,2, \ldots, \\
& y_{i}(t+1)=y_{i}(t)+u_{i}\left(t-l_{i}\right)-u_{i-1}(t), \quad i=1,2, \ldots,
\end{aligned}
$$

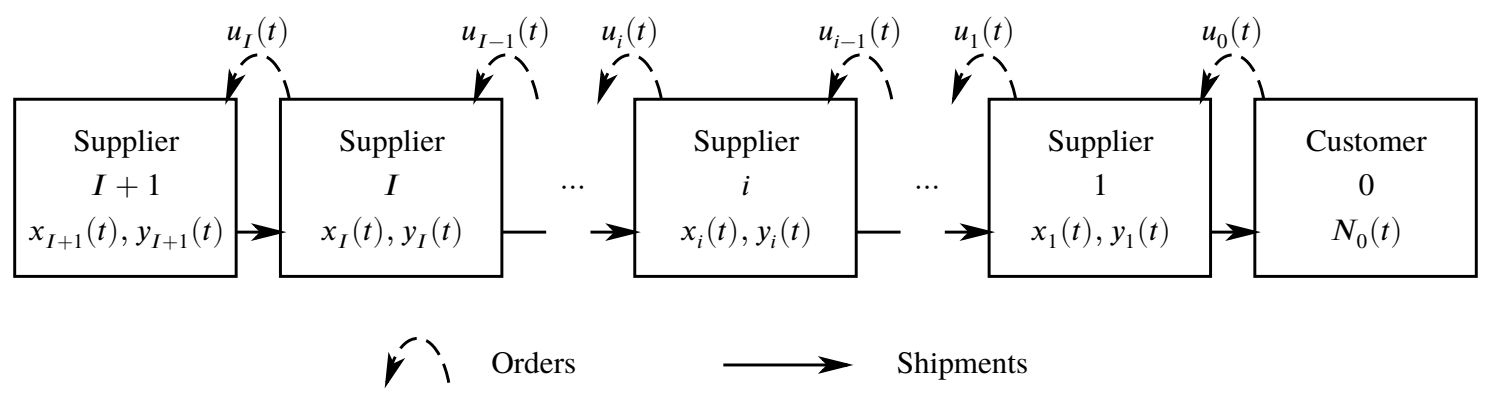

Figure 1: A serial supply chain. 
Generally, the ordering policies are based on the complete information set $\mathscr{F}_{i}(t)$ of supply chains including the inventory information $x_{i}, y_{i}$ and orders $u_{i}$ before period $t$ (Ouyang and Daganzo, 2006a), i.e., $u_{i}(t)$ is a function determined by

$$
\begin{aligned}
\mathscr{F}_{i}(t)=\{ & x_{i}(t), x_{i}(t-1), \ldots, x_{i}(-\infty) \\
& y_{i}(t), y_{i}(t-1), \ldots, y_{i}(-\infty) ; \\
& \left.u_{i-1}(t), u_{i-1}(t-1), \ldots, u_{i-1}(-\infty)\right\}, \quad i=1,2, \ldots
\end{aligned}
$$

In this paper, ordering policies are assumed to be (i) proper, i.e., the inventories are invariant, regardless of the initial condition, when the orders received are constant over time; (ii) nonlinear and time-invariant (NLTI); i.e., $u_{i}(t)$ is a time-independent nonlinear function of the elements in $\mathscr{I}(t)$. Define shift operator $\Delta$ as $\Delta^{k} u_{i}(t)=u_{i}(t-k), k=0,1, \ldots$, and the desired order $e_{i}(t)$ for supplier $i$ (Ouyang and Daganzo, 2006a), i.e.,

$$
e_{i}(t)=\gamma_{i}+P_{i}(\Delta) x_{i}(t)+Q_{i}(\Delta) y_{i}(t)+S_{i}(\Delta) u_{i-1}(t-1), \quad i=1,2, \ldots,
$$

where $\gamma_{i}$ is a constant real number, and $P_{i}(\Delta), Q_{i}(\Delta)$, and $S_{i}(\Delta)$ are polynomials with real coefficients. A general NLTI ordering policy can be expressed as follows:

$$
u_{i}(t)=N_{i}\left(e_{i}(t)\right), \quad i=1,2, \ldots,
$$

where $N_{i}(\cdot)$ is a nonlinear function. In our analysis, we assume that $N_{i}$ satisfies the following properties:

(i) $N_{i}(e)$ is nonnegative and monotonically increasing over $e \in \mathbb{R}$, i.e., ordered items cannot be returned, and the actual order quantity should be positively correlated with the desired order.

(ii) $N_{i}(e)$ is Lipschitz continuous, i.e., there exists a scalar $K_{i} \in \mathbb{R}_{+}$such that $\left|N_{i}\left(e_{1}\right)-N_{i}\left(e_{2}\right)\right| \leq$ $K_{i}\left|e_{1}-e_{2}\right|$ for any $e_{1}, e_{2} \in \mathbb{R}$.

Polynomials $P_{i}(\Delta), Q_{i}(\Delta)$, and $S_{i}(\Delta)$ reflect how the inventory and order history influence the desired order $e_{i}(t)$. Any possible NLTI ordering policies can be represented by a specific form of $N_{i}(\cdot), P_{i}(\Delta)$, $Q_{i}(\Delta)$, and $S_{i}(\Delta)$. This can be illustrated by the following example.

Example 1. (Order-up-to) If the fixed ordering cost equals zero, an order-up-to policy is optimal for each supplier. If the order-up-to level is forecasted as the moving average (M.A.) of the demand sequence $\left\{u_{i-1}\right\}$ (Dejonckheere et al., 2003), such a policy can be represented by (3) and (4) with $\gamma_{i}=0, P_{i}(\Delta)=-1$, $Q_{i}(\Delta)=0, S_{i}(\Delta)=\left(l_{i} / m\right) \sum_{k=0}^{m-1} \Delta^{k}=l_{i}\left(1-\Delta^{m}\right) /(m(1-\Delta))$, where $m$ is the number of periods used for demand forecasting, and $N_{i}\left(e_{i}(t)\right)=\max \left\{e_{i}(t), 0\right\}, \forall i$. If the order-up-to level is forecasted by exponential smoothing (E.S.) strategy (Dejonckheere et al., 2003), $S_{i}(\Delta)$ becomes $l_{i} \sum_{k=0}^{\infty} \alpha(1-\alpha)^{k} \Delta^{k}=$ $l_{i} \alpha /(1-(1-\alpha) \Delta)$ while all other parameters remain the same.

The properness tells that if the customer places a constant order of size $u^{\infty}$ and all the suppliers use proper policies, the system will reach a steady state (or equilibrium) where all the suppliers place the same order of size $u^{\infty}$ with the desired order $e_{i}^{\infty}$ and maintain the same inventories $x_{i}^{\infty}, y_{i}^{\infty}$. We assume without loss of generality that the system is in such equilibrium for all $t \leq 0$.

The formula of the equilibrium values can be derived by the observation that $u^{\infty}, x_{i}^{\infty}$, and $y_{i}^{\infty}$ naturally satisfy (1)-(4), i.e.,

$$
\begin{aligned}
e_{i}^{\infty} & =\gamma_{i}+P_{i}(1) x_{i}^{\infty}+Q_{i}(1) y_{i}^{\infty}+S_{i}(1) u^{\infty}, \quad i=1,2, \ldots \\
u^{\infty} & =N_{i}^{\infty}\left(e_{i}(t)\right), \quad i=1,2, \ldots
\end{aligned}
$$


where $N_{i}^{\infty}\left(e_{i}(t)\right)$ is the average of $N_{i}\left(e_{i}(t)\right)$ over $t$.

In bullwhip effect analysis, we focus on the variations of inventories and orders in system dynamics, expressed as the deviations from the equilibrium value, i.e., $\bar{x}_{i}(t)=x_{i}(t)-x_{i}^{\infty}, \bar{y}_{i}(t)=y_{i}(t)-y_{i}^{\infty}$, $\bar{e}_{i}(t)=e_{i}(t)-e_{i}^{\infty}$, and $\bar{u}_{i}(t)=u_{i}(t)-u^{\infty}$ for all $i$. Subtracting (5) from (3) and (6) from (4) yields

$$
\begin{aligned}
\bar{x}_{i}(t+1) & =\bar{x}_{i}(t)+\bar{u}_{i}(t)-\bar{u}_{i-1}(t), \quad i=1,2, \ldots, \\
\bar{y}_{i}(t+1) & =\bar{y}_{i}(t)+\bar{u}_{i}\left(t-l_{i}\right)-\bar{u}_{i-1}(t), \quad i=1,2, \ldots, \\
\bar{e}_{i}(t) & =P_{i}(\Delta) \bar{x}_{i}(t)+Q_{i}(\Delta) \bar{y}_{i}(t)+S_{i}(\Delta) \bar{u}_{i-1}(t-1), \quad i=1,2, \ldots, \\
\bar{u}_{i}(t) & =N_{i}\left(e_{i}(t)\right)-N_{i}^{\infty}\left(e_{i}(t)\right)=\bar{N}_{i}\left(\bar{e}_{i}(t), u^{\infty}\right), \quad i=1,2, \ldots,
\end{aligned}
$$

which is a homogeneous representation of the system dynamics.

\subsubsection{Frequency Domain Transformation}

Traditionally, supply chains are analyzed in the frequency domain by applying the $z$-transform since the optimal ordering policies (e.g., $(S, s)$ policy or order-up-to policy) are inherently based on discrete time (Vassian, 1955; Adelson, 1966; Elmaghraby, 1966; Deziel and Eilon, 1967). Denote the $z$-transforms ${ }^{1}$ of the discrete sequences by $X_{i}(z):=\mathscr{Z}\left\{\bar{x}_{i}(t)\right\}, Y_{i}(z):=\mathscr{Z}\left\{\bar{y}_{i}(t)\right\}, E_{i}(z):=\mathscr{Z}\left\{\bar{e}_{i}(t)\right\}$, and $U_{i}(z):=$ $\mathscr{Z}\left\{\bar{u}_{i}(t)\right\}$. Simple derivations show that $\mathscr{Z}\left\{P_{i}(\Delta) x_{i}(t)\right\}=P_{i}\left(z^{-1}\right) X_{i}(z), \mathscr{Z}\left\{Q_{i}(\Delta) y_{i}(t)\right\}=Q_{i}\left(z^{-1}\right) Y_{i}(z)$ and $\mathscr{Z}\left\{S_{i}(\Delta) u_{i}\right\}(t)=S_{i}\left(z^{-1}\right) U_{i}(z)$. Thus applying $z$-transform to (7) gives

$$
\begin{aligned}
z X_{i}(z) & =X_{i}(z)+U_{i}(z)-U_{i-1}(z)+z \bar{x}_{i}(0), \quad i=1,2, \ldots, \\
z Y_{i}(z) & =Y_{i}(z)+z^{-l_{i}} U_{i}(z)-U_{i-1}(z)+z \bar{y}_{i}(0), \quad i=1,2, \ldots, \\
E_{i}(z) & =P_{i}\left(z^{-1}\right) X_{i}(z)+Q_{i}\left(z^{-1}\right) Y_{i}(z)+z^{-1} S_{i}\left(z^{-1}\right) U_{i-1}(z), i=1,2, \ldots, \\
U_{i}(z) & =\mathscr{Z}\left\{\bar{N}_{i}\left(\bar{e}_{i}(t), u^{\infty}\right)\right\}:=\mathcal{N}_{i}\left(E_{i}(z)\right) .
\end{aligned}
$$

For demonstration convenience, we assume that the system begins with equilibrium state (i.e., $\bar{x}_{i}(0)=$ $\bar{y}_{i}(0)=0$ for any $\left.i=1,2 \ldots\right)$, and also define the cumulative order quantity $\bar{h}_{i}(t):=\sum_{\tau=-\infty}^{t} \bar{u}_{i}(\tau)$ and $H_{i}(z)=\mathscr{Z}\left\{\bar{h}_{i}(t)\right\}=\left(1-z^{-1}\right)^{-1} U_{i}(z)$, then the above equations can be simplified as the following:

$$
z E_{i}(z)=G_{i, 1}(z) H_{i}(z)+G_{i, 2} H_{i-1}(z),
$$

where

$$
\begin{aligned}
& G_{i, 1}(z)=P_{i}\left(z^{-1}\right)+Q_{i}\left(z^{-1}\right) z^{-l_{i}} \\
& G_{i, 2}(z)=-P_{i}\left(z^{-1}\right)-Q_{i}\left(z^{-1}\right)+S_{i}\left(z^{-1}\right)\left(1-z^{-1}\right) .
\end{aligned}
$$

\footnotetext{
${ }^{1}$ The $z$-transform of a given sequence $\{x(t)\}_{t=0}^{\infty}$ is given by $\mathscr{E}\{x(t)\}:=\sum_{t=0}^{\infty} x(t) z^{-t}$.
} 


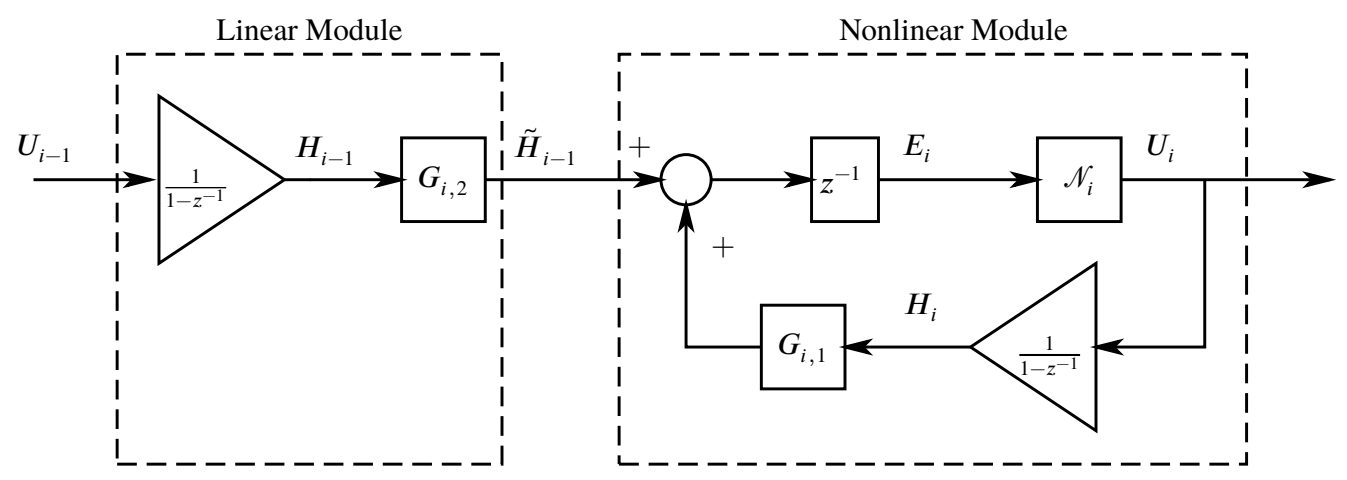

Figure 2: Flow diagram of the supply chain system.

Fig. 2 shows the block diagram for the above system dynamics. We observe that the system can be divided into two modules, i.e., a linear module including the integrator and $G_{i, 2}$, and a nonlinear module consisting of the remaining blocks. The orders from downstream $U_{i-1}$ first go through the linear module, gained by the integrator and $G_{i, 2}$, and then pass the nonlinear module and turn into $U_{i}$. Since we are interested in the magnitudes of those sequences, in the rest of this paper, we use $|\cdot|$ to denote the amplitude of the frequency component at its corresponding sequence's fundamental frequency.

\subsection{Fluctuation Propagation Analysis}

In this section, we will propose an analytical approach to quantify the fluctuation characteristics of the bullwhip effect for our general class of nonlinear ordering policies. This approach is based on the $z$-transform describing function method for fluctuation responses of a discrete nonlinear system (Vander Velde, 1968). The fluctuation characteristics quantitatively describe if and how small fluctuations in the customer demand are amplified into fully-grown fluctuations after several stages of suppliers.

\subsubsection{Describing Function Analysis}

Describing function analysis normally requires that the input signals are either sinusoidal or dominated by low frequency components. In supply chains, the order sequences $U_{i}$ often contain high frequency components, while $H_{i}$ is more likely dominated by low frequency components since the embedded integrator can serve as a low-pass filter (Blaquiere, 2012). Based on this observation, we equivalently transform the flow diagram Fig. 2 into Fig. 3, focusing on the analysis of $H_{i}$ instead of $U_{i}$ so that the describing function method can be applied and update the partition of linear and nonlinear modules accordingly. Now, based on Fig. 3, for the linear module, we define the linear gain as $R_{i}^{L}:=\left|\tilde{H}_{i-1}(z)\right| /\left|H_{i-1}(z)\right|=\left|G_{i, 2}(z)\right|$. Similarly, for the nonlinear module, the gain is denoted by $R_{i}^{N}:=\left|H_{i}(z)\right| /\left|\tilde{H}_{i-1}(z)\right|$.

If the input $\overline{\mathbf{u}}_{i-1}$ to system dynamics (1)-(4) can be approximated by a discrete sinusoid with amplitude $\left|U_{i-1}(z)\right|$ and frequency $\omega$, then the output $\overline{\mathbf{u}}_{i}$ should maintain the same frequency as it is generated from $\overline{\mathbf{u}}_{i-1}$. The low-pass property of the integrator dictates that $\overline{\mathbf{h}}_{i}$ shall also preserve a sinusoidal shape (although the phase angle may be different from that of $\overline{\mathbf{h}}_{i-1}$ ). It follows from Fig. 3 that in the frequency-domain,

$$
H_{i}(z) \approx \tilde{\mathscr{N}}_{i}\left(\bar{e}_{i}(t), u^{\infty}, \omega\right) \cdot \frac{1}{1-z^{-1}} \cdot E_{i}(z)
$$




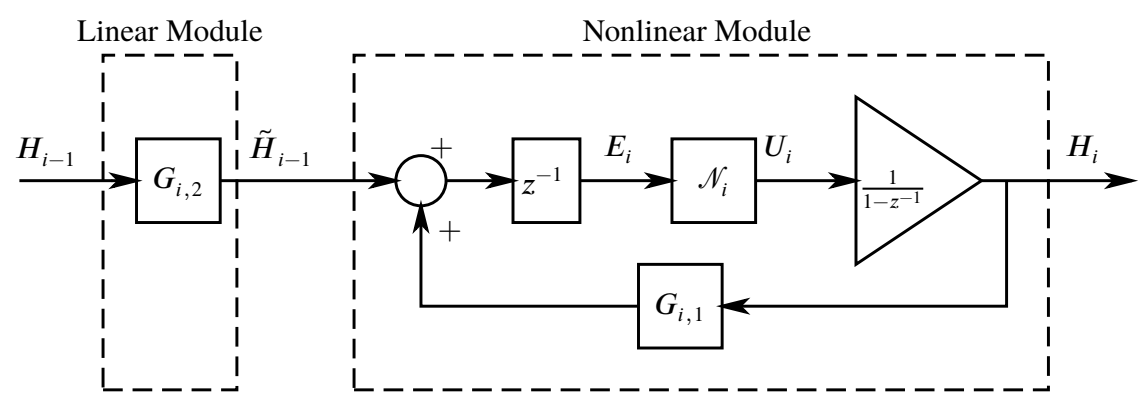

Figure 3: Modified flow diagram of the supply chain system.

where $\tilde{\mathscr{N}}_{i}\left(\bar{e}_{i}(t)\right)$ is a nonlinear coefficient defined as follows:

$$
\tilde{\mathscr{N}}_{i}\left(\bar{e}_{i}(t), u^{\infty}, \omega\right)=\frac{j \omega}{\pi\left|E_{i}(z)\right|} \int_{0}^{2 \pi / \omega} \bar{N}_{i}\left(\bar{e}_{i}(t), u^{\infty}\right) \exp (-j \omega t) \mathrm{d} t,
$$

which is used to linearly approximate $\mathcal{N}_{i}$. Substituting (12) into (9) gives

$$
H_{i-1}(z) \approx G_{i, 2}(z)^{-1} E_{i}(z)\left(z-G_{i, 1}(z) \cdot \tilde{\mathscr{N}}_{i}\left(\bar{e}_{i}(t), u^{\infty}, \omega\right) \cdot \frac{1}{1-z^{-1}}\right),
$$

which together with (12) yields

$$
\left\{\begin{aligned}
\left|H_{i-1}(z)\right| & \approx\left|G_{i, 2}(z)\right|^{-1} \cdot\left|E_{i}(z)\right| \cdot\left|z-G_{i, 1}(z) \cdot \tilde{\mathscr{N}}_{i}\left(\bar{e}_{i}(t), u^{\infty}, \omega\right) \cdot \frac{1}{1-z^{-1}}\right|, \\
\left|H_{i}(z)\right| & \approx\left|E_{i}(z)\right| \cdot\left|\tilde{\mathscr{N}}_{i}\left(\bar{e}_{i}(t), u^{\infty}, \omega\right) \cdot \frac{1}{1-z^{-1}}\right|
\end{aligned}\right.
$$

Formula (14) can be used to determine output $\left|H_{i}(z)\right|$ for any given input $\left|H_{i-1}(z)\right|$. Since $H_{i}(z)$ is simply the cumulation of $U_{i}(z)$, we can then obtain $\left|U_{i}(z)\right|$ from $\left|U_{i-1}(z)\right|$. If each supplier in the supply chain shares the same ordering policy, we can apply this recursive relationship to all supplier stages iteratively to obtain the amplitudes of the order sequences for all upstream suppliers from any given $\left|U_{i}(z)\right|$.

Moreover, with the help of (14), the test of bullwhip effect under a nonlinear ordering policy can be derived. Suppose the fundamental frequency of the customer order sequence is $\omega$, i.e., $z=e^{j \omega}$, we can conclude that the bullwhip effect arises if and only if

$$
\left|\frac{H_{I}\left(e^{j \omega}\right)}{H_{0}\left(e^{j \omega}\right)}\right|=\prod_{i=1}^{I} \frac{\left|G_{i, 2}\left(e^{j \omega}\right) \cdot \tilde{\mathscr{N}}_{i}\left(\bar{e}_{i}(t), u^{\infty}, \omega\right)\right|}{\left|\left(e^{j \omega}-1\right)-G_{i, 1}\left(e^{j \omega}\right) \cdot \tilde{N}_{i}\left(\bar{e}_{i}(t), u^{\infty}, \omega\right)\right|}>1 .
$$

This test is complex but applicable to any nonlinear ordering policy. In Section 3.1 we show that by specifying $N_{i}$, formula (15) can be simplified.

In reality, ordering policies normally involve physical constraints, and hence, $N_{i}$ is usually bounded. When (15) holds, since the discrete sequences $\overline{\mathbf{u}}_{i}$ have a finite mean, i.e., zero, the magnitude of $\left|U_{i}(z)\right|, \forall i$, has to be bounded as well. Therefore, when $i \rightarrow \infty$, if we further assume that the magnitude $\left|U_{i}(z)\right|$ is increasing monotonically with $i$, it follows from monotone convergence theorem that $\left|U_{i}(z)\right|$ will converge to one finite value, i.e., there exists a steady state such that the amplitudes of order variations become stable after several supply chain stages. Let $s$ be the amount of time shift for order sequences between adjacent 
suppliers. Theoretically, the steady state in the supply chain system can be expressed as: $\overline{\mathbf{u}}_{i-1}$ is of the same waveform as $\overline{\mathbf{u}}_{i}$, with only a time shift of $s$ time periods. The time shift is negative since the upstream orders $U_{i}(z)$ is determined by the history of downstream orders $U_{i-1}(z)$. It follows that $U_{i}(z)=z^{s} U_{i-1}(z)$, and thus $H_{i}(z)=U_{i}(z)\left(1-z^{-1}\right)^{-1}=z^{s} H_{i-1}(z)$. Substituting them into (9) and (8) yields

$$
U_{i}(z)=\widetilde{N}_{i}\left(U_{i}(z) \cdot z^{-1}\left(\frac{1-z^{-s}}{1-z^{-1}} P_{i}\left(z^{-1}\right)+\frac{z^{-l_{i}}-z^{-s}}{1-z^{-1}} Q_{i}\left(z^{-1}\right)+z^{-s} S_{i}\left(z^{-1}\right)\right)\right), \quad i \rightarrow \infty
$$

for some $s \in \mathbb{Z}_{-}$, which is the condition for the waveform $U_{i}(z)$ to be in steady state for generalized ordering policies. It shall be noted, nevertheless, that in case $\left|U_{i}(z)\right|$ does not monotonically increase with $i$, it may not converge but rather oscillate between multiple values.

\subsubsection{Approximation Refinement}

The DFA developed in the preceding section uses a quasi-linear approximation of the nonlinear operator, i.e., both inputs and outputs of the nonlinear operator are assumed to be sinusoids. In most cases, this approximation yields accurate predictions since the low pass filter ensures that the inputs of nonlinear module are sinusoidal or at least dominated by low frequency components. However, in some situations, the nonlinear module may produce high frequency components that cannot be eliminated by the low-pass filter. Specifically, any solution to (16) cannot be accurately approximately as sinusoids by (14) if the solution $U_{i}(z), i \rightarrow \infty$, is a scaled and shifted sampling function ${ }^{2}$. The following proposition illustrates such situations.

Proposition 1. The order sequences may converge to a scaled and shifted sampling function with magnitude $a$ and period $T$ if the following equation holds.

$$
\frac{a}{1-z^{-T}}=\mathcal{N}_{i}\left(\frac{a z^{-1}}{1-z^{-T}}\left(\frac{1-z^{-s}}{1-z^{-1}} P_{i}\left(z^{-1}\right)+\frac{z^{-l_{i}}-z^{-s}}{1-z^{-1}} Q_{i}\left(z^{-1}\right)+z^{-s} S_{i}\left(z^{-1}\right)\right)\right), \quad i \rightarrow \infty
$$

Proof. By definition, the $z$-transform of the input $\mathbf{u}_{i-1}, i \rightarrow \infty$, is

$$
U_{i-1}(z)=a \sum_{k=0}^{\infty} \mathscr{Z}\{\delta(t-k T)\}=a \sum_{k=0}^{\infty} z^{-k T}=\frac{a}{\left(1-z^{-T}\right)} .
$$

Substituting this into (16) yields the conclusion.

Intuitively, this proposition can be explained as follows. First, note that the average order quantity $u^{\infty}$ is invariant (due to conservation law) while the amplitudes in the time domain is increasing. Therefore, the widths of waves in the order sequences shrink to impulses in the steady state as $i \rightarrow \infty$. Finally, the discreteness of the sequence implies that the amplitudes have to be bounded. Moreover, such result reasonably explains the fact in empirical supply chains where manufacturers tend to observe longer intervals between orders and larger ordering quantities than retailers.

On the other hand, Proposition 1 implies that the describing-function-based approximation (14) need to be adjusted in order to cover those asymptotic situations (i.e., the order sequences are no longer sinusoidal for $i \rightarrow \infty)$. To quantity the amplitudes, define $U_{\max }:=\lim _{i \rightarrow \infty}\left|U_{i}(z)\right|, H_{\max }:=\lim _{i \rightarrow \infty}\left|H_{i}(z)\right|$ and $E_{\max }:=\lim _{i \rightarrow \infty}\left|E_{i}(z)\right|$ as the amplitudes in steady state for signals $\overline{\mathbf{u}}_{i}, \overline{\mathbf{h}}_{i}$, and $\overline{\mathbf{e}}_{i}$ respectively. The result is concluded in the following proposition.

\footnotetext{
${ }^{2}$ The sampling function is a periodic Schwartz distribution constituted by Dirac delta functions (impulses), i.e., $\sum_{k=-\infty}^{\infty} \delta(t-$ $k T)$, where $T$ is the period (Córdoba, 1989).
} 
Proposition 2. (Asymptotic fluctuation magnitude in the steady state) In the steady state, when the waveform of order sequences is from a scaled and shifted sampling function, the magnitudes of all suppliers' order fluctuations are proportional to the size of the nominal order $u^{\infty}$ and inversely proportional to the frequency $\omega$ of the customer demand.

Proof. As illustrated in Fig. 4, in the steady state $(i \rightarrow \infty)$, order sequences $\mathbf{u}_{i}$ are from the sampling function; cumulative orders $\mathbf{h}_{i}$ are stepwise and $\overline{\mathbf{h}}_{i}$ (obtained by eliminating nominal parts from $\mathbf{h}_{i}$ ) are sawtooth waves. Suppose the magnitudes of suppliers' order fluctuations in the steady state equals $a$, it follows from the $z$ transform that $U_{\max }=2 a / T, H_{\max }=a / \pi$. Also, as discussed in Section 2.1.1, the slope of stepwise curve $\mathbf{h}_{i-1}$ should be the equilibrium constant $u^{\infty}$, i.e., $u^{\infty}=a / T$. Since $T=2 \pi / \omega$, we see that

$$
U_{\max }=2 u^{\infty}, \quad H_{\max }=2 u^{\infty} / \omega, \quad a=2 \pi u^{\infty} / \omega .
$$
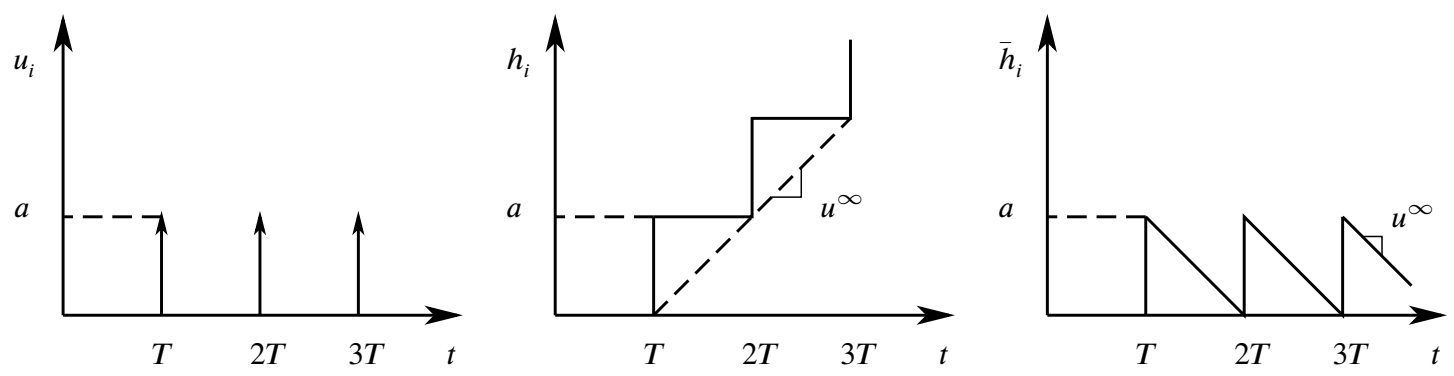

Figure 4: Illustration of the asymptotic order sequences in the steady state.

The conclusion follows (18). Note too that the magnitudes of the fundamental frequency component of the steady order sequences $U_{\max }$ is twice the magnitude of the nominal order quantity $u^{\infty}$, and it is not influenced by the frequency value $\omega$.

Proposition 2 provides connections between system parameters and asymptotic properties of the order fluctuations. Meanwhile, it shows that the demand forecasting method (e.g., M.A. or E.S.) would have no effects on these asymptotic properties. This result directly leads to conditions under which the bullwhip effect may not exist: (i) the average demand $u^{\infty}$ is sufficiently low; (ii) the frequency of the customer demand fluctuations are sufficiently high. In correspondence, marketing/management strategies may also be developed against the bullwhip effect; e.g., by reducing the average demand per order $u^{\infty}$, or by increasing the frequency of customer orders.

Finally, the next proposition provides a refined quantification of the asymptotic steady state.

Proposition 3. (Refinement law) When the waveform of order sequences in the steady state is from a scaled and shifted sampling function, for all $i=1,2, \ldots$, the amplitudes of suppliers' cumulative and desired order sequences satisfy the following equations.

$$
\left\{\begin{aligned}
&\left|H_{i-1}(z)\right| \approx\left(\varphi_{11}(i)\left|G_{i, 2}(z)\right|^{-1} \cdot\left|z-G_{i, 1}(z) \cdot \tilde{\mathcal{N}}_{i}\left(\bar{e}_{i}(t), u^{\infty}, \omega\right) \cdot \frac{1}{1-z^{-1}}\right|\right. \\
&\left.+\varphi_{12}(i) \frac{2 u^{\infty}}{\omega E_{\max }}\right)\left|E_{i}(z)\right|, \\
&\left|H_{i}(z)\right| \approx\left(\varphi_{21}(i) \cdot\left|\tilde{\mathcal{N}}_{i}\left(\bar{e}_{i}(t), u^{\infty}, \omega\right) \cdot \frac{1}{1-z^{-1}}\right|+\varphi_{22}(i) \frac{2 u^{\infty}}{\omega E_{\max }}\right)\left|E_{i}(z)\right| . \\
& i=1,2, \ldots
\end{aligned}\right.
$$


where $\varphi_{j k}(i), j, k=1,2$ are refinement coefficients satisfying

$$
\begin{array}{lll}
\varphi_{j 1}(i) \rightarrow 1, & \varphi_{j 2}(i) \rightarrow 0 & \text { for } i \rightarrow 0 \\
\varphi_{j 1}(i) \rightarrow 0, & \varphi_{j 2}(i) \rightarrow 1 & \text { for } i \rightarrow \infty
\end{array}
$$

Proof. See Appendix A.1.

The coefficients $\varphi_{j k}(i)$ can be chosen arbitrarily; e.g., by proper interpolation between $\left|U_{0}(z)\right|$ and $U_{\max }$. In the next section, we will propose a specific way of choosing these coefficients.

\section{Special Case: Order-up-to Policy}

The modeling framework proposed in Section 2.2 is general and covers a wide range of ordering policies (i.e., with various linear operators $G_{i, 1}, G_{i, 2}$ and nonlinear function $N_{i}$ ). For illustration purposes, we now focus on a widely used nonlinear ordering policy, i.e., the order-up-to policy as in Example 1 with $P_{i}\left(z^{-1}\right)=$ $-1, Q_{i}\left(z^{-1}\right)=0$. Moreover, we only consider homogenous supply chains, i.e., all suppliers in a multiechelon supply chain share this same ordering policy, and thus the subscript $i$ in $P_{i}\left(z^{-1}\right), Q_{i}\left(z^{-1}\right), S_{i}\left(z^{-1}\right)$ and $N_{i}$ are omitted in the rest of this section.

Before analyzing the bullwhip effect under the order-up-to policy, it is also necessary to verify the existence of the bullwhip effect from (15). Since $N\left(e_{i}(t)\right)=\max \left\{e_{i}(t), 0\right\}$, when testing bullwhip effect with slightly fluctuating customer demand such that $N\left(e_{0}(t)\right)=e_{0}(t)$, it follows from $(13)$ that $\tilde{\mathcal{N}}\left(\bar{e}_{i}(t), u^{\infty}, \omega\right)=$ 1 and (15) is exactly the same as the test for a linear time-invariant (LTI) supply chain. Therefore, the simplified formula of (15) for the order-up-to policy in a homogenous chain is

$$
\left|z^{-1} G_{i, 2}(z)\right|=\left|z^{-1}\left(1+S\left(z^{-1}\right)\left(1-z^{-1}\right)\right)\right|>1 .
$$

This test further generalizes the corresponding results in Ouyang and Daganzo (2006a). In the rest of this section, we assume that condition (20) holds and hence the bullwhip effect exists.

\subsection{Analytical Predictions}

Since the order-up-to policy is piece-wise linear with a simple truncation, it performs the same as a linear ordering policy if the desired order quantity is positive. To this end, the analysis is conducted in the following two aspects.

(i) Linear Phase. When the desired order quantity is positive, lying in the linear segment of the nonlinear function $N$, the amplitudes of order sequences should grow exponentially along the chain, i.e., the prediction by our framework should be consistent with the linear theory (Ouyang and Daganzo, 2006a) if eliminated the assumption for nonlinearity;

(ii) Nonlinear Phase. When the desired order quantity is negative, considering the influence of nonlinear function $N$, the amplitudes of order sequences should be bounded after several stages along the chain, i.e., our prediction realistically matches the empirical phenomena observed by Cachon et al. (2007).

Following the standard approach for studying the linear phase (Haykin and Van Veen, 2007), we have the following lemma. 
Lemma 1. By using moving average or exponential smoothing to forecast the order-up-to level, the amplitudes of the cumulative order sequences $\overline{\mathbf{h}}_{i-1}$ always increase through the linear module, i.e., the linear gain satisfies $R_{i}^{L}>1$.

Proof. This is a direct implication of (20) since

$$
R_{i}^{L}=\left|G_{i, 2}(z)\right|=\left|1+S\left(z^{-1}\right)\left(1-z^{-1}\right)\right|=\left|z^{-1}\left(1+S\left(z^{-1}\right)\left(1-z^{-1}\right)\right)\right| .
$$

Our second step is to show that the nonlinear module effects the fluctuations in the opposite direction. For simplicity we assume that $e_{i}(t)=\left|E_{i}(z)\right| \sin (\omega t)+e_{i}^{\infty}$, which does not include a phase angle since we can always shift the time axis to remove it. Observing that the nonlinear function $N_{i}\left(e_{i}(t)\right)=\max \left\{e_{i}(t), 0\right\}$ is defined in the time domain, define the critical phase angle

$$
\theta_{i}:=\arg _{\theta_{i} \in(-\pi / 2, \pi / 2]}\left|E_{i}(z)\right| \sin \left(-\theta_{i}\right)+e_{i}^{\infty}=0,
$$

which corresponds to the time $t_{i}=\theta_{i} / \omega$ when $e_{i}(t)$ is first truncated by $N_{i}$. Also, define the critical stage

$$
i_{0}:=\arg _{i_{0} \in \mathbb{Z}}\left|E_{i_{0}}(z)\right|=e_{i_{0}}^{\infty},
$$

where $e_{i}(t)$ first gets truncated. Note that here $\theta_{i_{0}}=\pi / 2$. Then we have the following result.

Lemma 2. If each supplier in the supply chain shares the same order-up-to policy, the amplitude of $\tilde{\mathbf{h}}_{i-1}$ will not be enlarged through the nonlinear module, i.e., $R_{i}^{N} \leq 1$. Specifically, $(i)$ in linear phase, $R_{i}^{N}=1$, and (ii) in nonlinear phase, $R_{i}^{N}<1$.

Proof. See Appendix A.2.

Moreover, we can directly obtain the following corollary from Lemma 2.

Corollary 1. The specific form of (14) for order-up-to policy is

$$
\left\{\begin{aligned}
\left|H_{i-1}(z)\right| & \approx\left(R_{i}^{L}\right)^{-1}\left(\left(\frac{g_{1}\left(\theta_{i}\right)}{2 \sin (\omega / 2)}\right)^{2}+\left(1-g_{1}\left(\theta_{i}\right)\right)\right)^{1 / 2} \cdot\left|E_{i}(z)\right|, \\
\left|H_{i}(z)\right| & \approx \frac{g_{i}\left(\theta_{i}\right)}{2 \sin (\omega / 2)} \cdot\left|E_{i}(z)\right| .
\end{aligned}\right.
$$

where

$$
\begin{aligned}
& g_{1}\left(\theta_{i}\right)=\frac{1}{2}+\frac{\theta_{i}}{\pi}+\frac{\sin \left(2 \theta_{i}\right)}{2 \pi} \\
& g_{2}\left(\theta_{i}\right)=\frac{1}{\pi}\left(\cos \theta_{i}+\theta_{i} \sin \theta_{i}\right)+\frac{1}{2} \sin \theta_{i}
\end{aligned}
$$

and $\left|E_{i}(z)\right|=u^{\infty} / g_{2}\left(\theta_{i}\right)$.

Proof. See Appendix A.2.

With the help of Lemmas 1 and 2, we can obtain the following analytical results for the bullwhip effect. 
Proposition 4. (Bounded growth of the bullwhip effect) If $R_{i}^{L}>1$, the amplitudes of the order sequences will grow exponentially when $i \leq i_{0}$ and then tend to stabilize and finally reach a bounded steady state, where the following equation holds.

$$
R_{i}^{L} R_{i}^{N}=1, \quad i \rightarrow \infty .
$$

If $R_{i}^{L}=1$, the amplitudes of the order sequences should be invariant along the chain, i.e., there is no bullwhip effect.

Proof. See Appendix A.3.

In Lemma 2 we show that $R_{i}^{N}=1$ and thus $R_{i}^{L} R_{i}^{N}>1$ in the linear phase, which is consistent with the findings in Ouyang and Daganzo (2006a) with linearized policies. However, in the nonlinear phase, Lemma 2 indicates that we may have $R_{i}^{N}<1$ and it will be possible that $R_{i}^{L} R_{i}^{N}=1$.

Proposition 4 shows that in supply chain, the amplitude of the fluctuations is bounded under nonlinear ordering policies, which is consistent with the empirical phenomena observed by Cachon et al. (2007). Proposition 4 also implies that the forecasting methods have no effect on the asymptotic bullwhip effect "bound" (i.e., the maximum amplitude that can be achieved after multiple stages). Before that bound is reached, the amplitude growth largely depends on the magnitude of the linear gain, which definitely depends on the forecasting method. Note that the second conclusion in Proposition 4 provides another direction for possibly eliminating the bullwhip effect, i.e., reducing the linear gain $R_{i}^{L}=\left|G_{i, 2}(z)\right|$ to 1 . Intuitively, lower linear gain implies lower increasing rate before the amplitude reaches the bound, and hence $R_{i}^{L}=1$ implies that the amplitudes will never grow, i.e., the bullwhip effect does not arise. To achieve this goal, observe that $G_{i, 2}(z)$ contains $S_{i}\left(z^{-1}\right)$ which is related to the forecasting method. Therefore, with sufficient information about customer demand, by adjusting the forecasting parameters one can control the value of $R_{i}^{L}$. For instance, the linear gain $R_{i}^{L}=1$ if the number of past periods used in M.A. forecasting is chosen to be $m=2 \pi / \omega$.

Finally, we introduce the following proposition to show that for certain order-up-to policies, the order sequences in the steady state are from a scaled and shifted sampling function.

Proposition 5. The order sequences of the upstream suppliers in the steady state (when $i \rightarrow \infty$ ) are from a scaled and shifted sampling function with the same amplitude and periodicity, i.e., the order sequences of the upstream suppliers contain non-negligible high frequency components, as long as the coefficients in $S_{i}(\Delta)$ are nonnegative and monotonically decreasing in value with respect to the degrees of their corresponding exponent terms.

Proof. See Appendix A.4.

As a consequence, using the method introduced in Section 2.2.2, the following laws are derived as a refinement of the prediction under order-up-to policies. Since the system is in linear phase when $i<i_{0}$, it can be concluded that the refinement is only necessary when $i \geq i_{0}$. We will use a linear interpolation between $\left|H_{i_{0}}(z)\right|$ and $H_{\max }$ for the refinement, i.e.,

$$
\varphi_{11}(i)=\varphi_{21}=1-\lambda(i), \quad \varphi_{12}(i)=\varphi_{22}=\lambda(i)
$$

where

$$
\lambda(i)=\frac{R_{i}^{L}\left|H_{i-1}(z)\right| \cdot\left|H_{i_{0}}(z)\right|^{-1}-1}{R_{i}^{L} H_{\max } \cdot\left|H_{i_{0}}(z)\right|^{-1}-1} .
$$


Note that $H_{\max }=2 u^{\infty} / \omega$ and Appendix A.1 implies that

$$
E_{\max }=2 u^{\infty}+\frac{\omega}{\pi} \int_{0}^{2 \pi / \omega} F\left(2 \pi u^{\infty} / \omega, \tau\right) \sin (\omega \tau) \mathrm{d} \tau,
$$

where $F(h, \tau)=-h \cdot 1_{\tau \in[\bmod (m, 2 \pi / \omega), 2 \pi / \omega]}$ for moving average and $F(h, \tau) \approx \alpha h\left((1-\alpha)^{\tau}-1\right)$ for exponential smoothing, respectively. Now (19), (21) and (23) form a complete set of equations for an accurate prediction. They will be used in the following numerical experiments.

\subsection{Numerical Experiments}

In this subsection we will conduct numerical experiments on the order-up-to policy where the order-up-to level is forecasted by either moving average or exponential smoothing methods (see Example 1). In both cases, the system parameters are chosen as follows: $\omega=0.02 \pi \sim 0.2 \pi, C=u^{\infty}=10$ and $l_{i}=15$ for any $i$. The customer demand is a sinusoid added with a bias, i.e., $u_{0}(t)=A_{0} \sin (\omega t)+C$, where $A_{0}=\left|U_{0}(z)\right|=$ $5 \omega$. For moving average, we set $m=10$ for the number of past periods used to forecast the demand, and for exponential smoothing, we set $\alpha=1 / 10$ for the smoothing weight. We simulate a supply chain with 10 stages for 500 time steps (i.e., weeks) in 10 years.

For each supplier, we calculate the cumulative orders $\left\{\overline{\mathbf{h}}_{i}\right\}_{\forall i}$ from simulation and plot the magnitudes $\left\{\left|H_{i}(z)\right|\right\}_{\forall i}$ of their fundamental components from the Fourier transform as the solid curves in Fig. 5. Then we apply the proposed mathematical framework to predict the magnitudes with the same input $\mathbf{u}_{0}$, which is plotted as the dashed curves. We see that the analytical predictions are very close to the simulation results. It is also shown that the amplitudes tend to be bounded after just a few supplier stages, which conform with Proposition 4.

It shall be noted from Fig. 5a-5b that the simulated amplitude bounds under those two forecasting methods are the same, $159.2=H_{\max }=2 u^{\infty} / \omega=2 \times 10 /(0.04 \pi)$. This is consistent with the prediction in Proposition 2. In contrast, under linear policies, the amplitudes will grow exponentially, which is far different from those obtained from simulations. From Fig. 5c, we see that the waveform of supplier 3 is still sinusoidal and not truncated by $N_{i}$. Therefore, supplier 2 is in linear phase and thus the amplitude should be $\left|U_{i}(z)\right|=$ $\left|H_{i}(z)\right| \omega=76.9 \times 0.04 \pi=9.7$ which consistent with Fig. $5 c\left(19.7-u^{\infty}=9.7\right)$. Similar observations can be made in Fig. 5d for supplier 10. On the other hand, Fig. 5a-5b show that supplier 10 is in the steady state under both forecasting methods, and thus Fig. $5 \mathrm{c}-5 \mathrm{~d}$ verify Propositions 5 and 2 by showing that the order sequences are indeed from a sampling function with magnitudes $a=2 \pi u^{\infty} / \omega=2 \pi \times 10 /(0.04 \pi)=500$, whose value again does not depend on the forecasting method.

While changing $\omega$, we calculate the mean squared error (MSE) between the predicted and simulated amplifications. The results are shown in Fig. 6a. It can be seen that the proposed analytical framework can accurately predict the fluctuations under different settings. In addition, the error is decreasing as the frequency increases, which is intuitive since high frequency components are having a more significant effect on the amplitudes while the system inputs have a lower fundamental frequency. For fluctuation propagation shown in Fig. 6b, it can be observed that the maximum magnitude $H_{\max }$ is decreasing as the demand frequency $\omega$ increases, and this phenomenon has been proved in Proposition 2. Moreover, when $\omega=0.2 \pi$, for moving average forecasting, we have $R_{i}^{L}=1$ for any $i$. Proposition 4 implies that there will be no bullwhip effect (i.e., each supplier has the same magnitudes of order sequences). This prediction is observed in Fig. $6 \mathrm{~b}$.

To further verify Proposition 4, Fig. 7 shows the simulated bullwhip effect growth under moving average and exponential smoothing policies with different forecasting parameters (all other parameters remain the same). For moving average, we alter $T$, while for exponential smoothing, we alter $\alpha$. It can be seen that with 


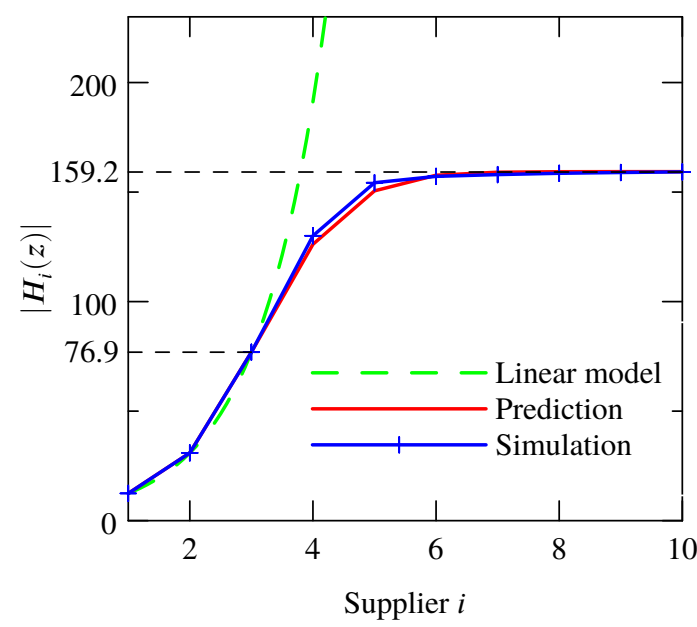

(a)

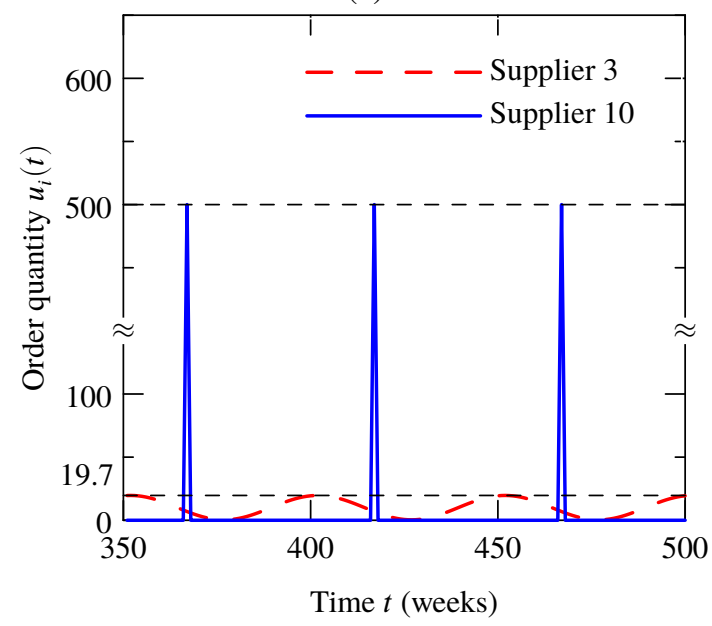

(c)

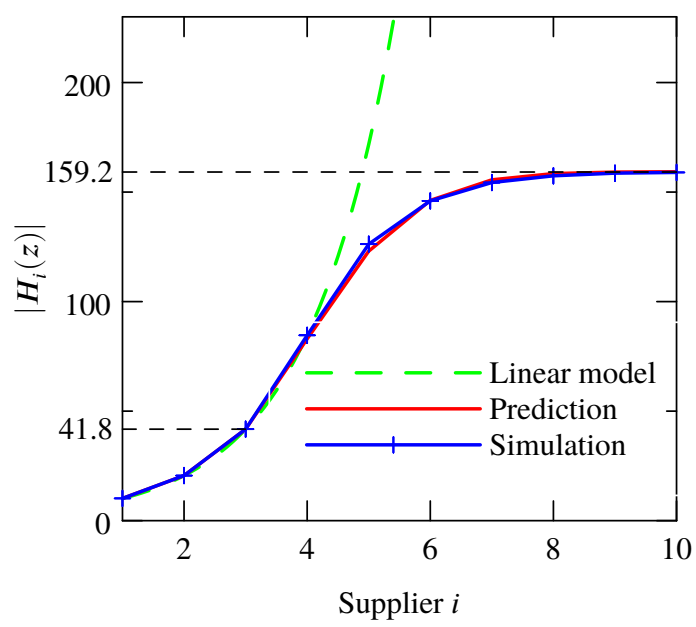

(b)

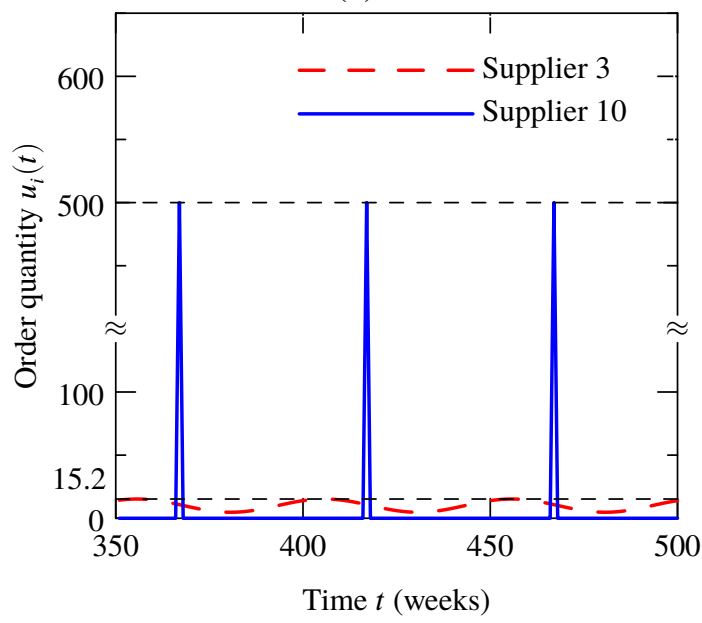

(d)

Figure 5: Comparisons of the bullwhip effect prediction under demand frequency $\omega=0.04 \pi$ and order-up-to policies with (a) moving average and (b) exponential smoothing forecasting methods. Subfigures (c) and (d) show the corresponding order sequences for supplier 3 and 10 under (a) and (b), respectively.

smaller $T$ or larger $\alpha$, the amplitudes grow faster and reach the amplitude bound earlier. However, regardless of these parameter values, the curves in each case converge to the same growth bound eventually.

\section{Conclusion and Discussion}

This paper proposes a mathematical framework that can be used to analyze the bullwhip effect in multiechelon supply chains under a general class of nonlinear ordering policies. The supply chain is modeled as a cascading nonlinear control system, and the $z$-transform describing function method is applied to analyze the cumulative order sequences. A systematic framework is developed to analytically quantify and predict the magnitudes of order fluctuations across suppliers, and to provide managerial insights. By investigating the characteristics of the asymptotic magnitude of the bullwhip effect in the steady state, we propose a refinement 


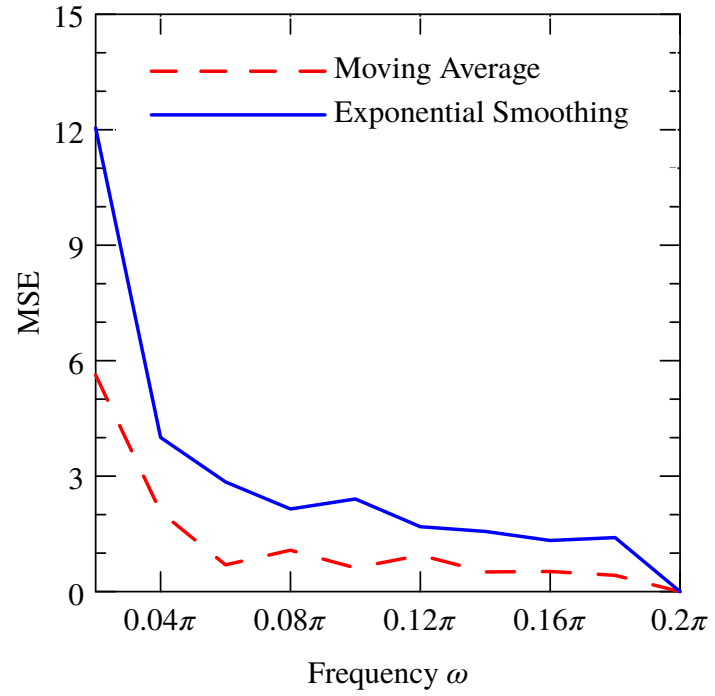

(a)

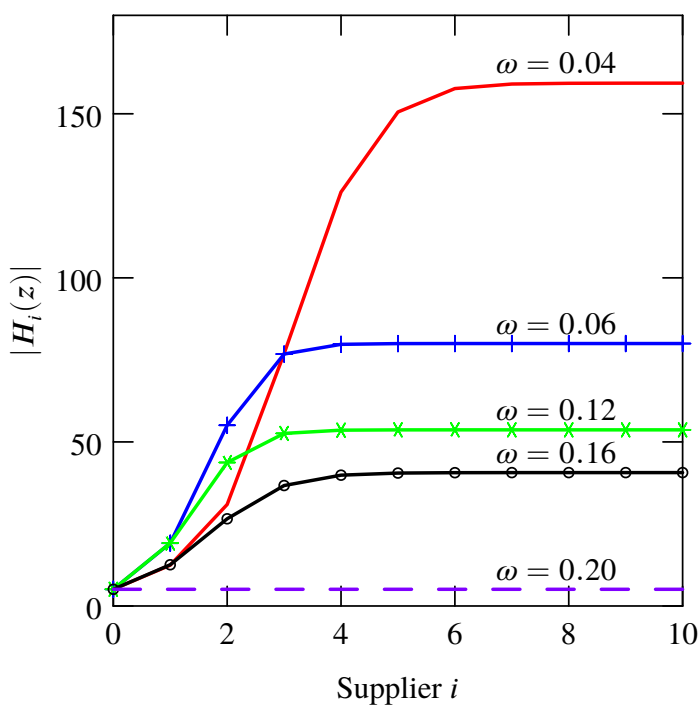

(b)

Figure 6: Impacts of customer demand frequency $\omega$ : (a) predicted and simulated order MSE ; (b) Order fluctuation propagation under moving average forecasting.

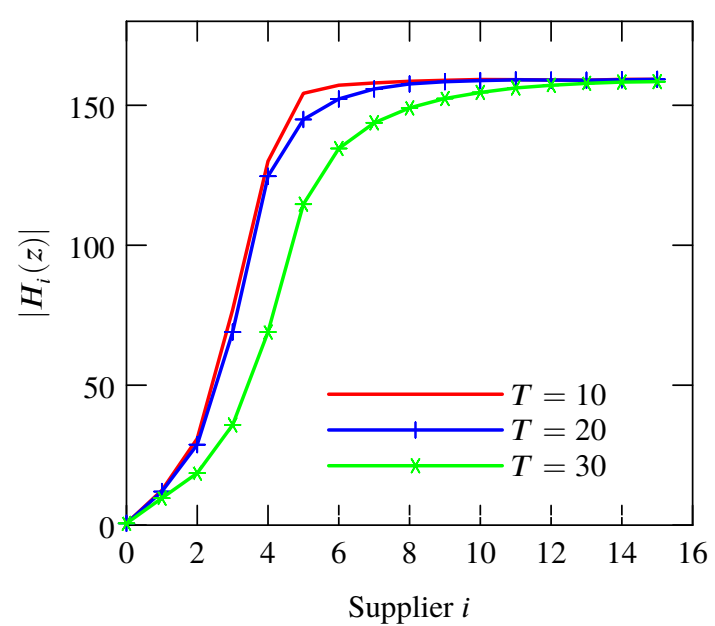

(a)

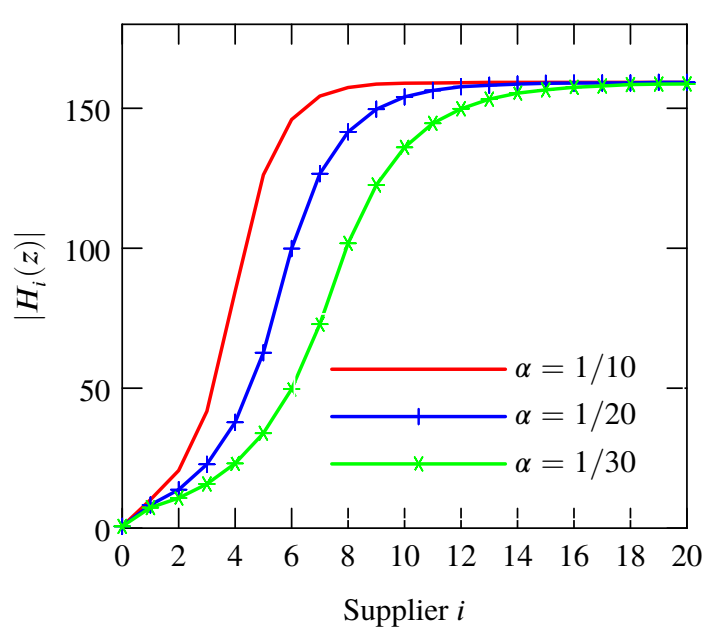

(b)

Figure 7: Impacts of forecasting method: (a) moving average with varying $T$; (b) exponential smoothing with varying $\alpha$. 
method to improve the accuracy of the standard describing function approach. Further analysis and numerical experiments show that the proposed modeling framework can accurately predict the amplitudes of order fluctuations for a wide range of ordering policies and any demand forecasting methods. Comparing with typical methods assuming linearized ordering policies, our method provides a more realistic characterization for the growth of order fluctuations.

Our framework can serve as the foundation for developing control strategies to effectively mitigate the bullwhip effect, possibly following similar ideas in Ouyang and Daganzo (2006b) and Ouyang (2007, 2014). Several interesting observations and conclusions are drawn from our analytical results. For example, it follows from Proposition 2 that high customer demand per order (or low ordering frequencies) may result in larger bullwhip effect among upstream suppliers. Based on this fact, supply chain managers may consider strategies and incentives that encourage the customers to lower the per-order demand and increase ordering frequency - although this may imply higher transaction/operation costs. In addition, Proposition 4 reveals another way to eliminate the bullwhip effect; i.e., by choosing proper forecasting methods and parameters such that the linear gain equals 1 . This strategy would be much more feasible to the managers since it only requires adjustments to the demand forecasting techniques.

This research can be extended in several directions. The supply chain is modeled as a single-input-singleoutput system (i.e., the actual order is a univariate function of the desired order; see Fig. 3). Such a set-up may fail to capture more complicated ordering policies (e.g., the $(S, s)$ policy). It will be appealing to extend our framework to multi-input-single-output systems (e.g., allowing the actual order as a multivariate function of not only the desired order but also other variables, such as inventory position). Second, the system input (i.e., the fluctuating component of the customer demand) is assumed to be sinusoids; it may also be ideal to further generalize this into any random processes, e.g., the Gaussian process. We are also interested in validating our modeling framework using empirical data from real-world supply chains.

\section{Acknowledgments}

This research was supported in part by the U.S. National Science Foundation via Grant CMMI-0748067. The first author thanks Professor Tamer Başar (UIUC) for his helpful comments at the early stage of this research.

\section{A Proofs}

\section{A.1 Proof for Proposition 3}

Proof. (9) implies that $E_{i}(z)=U_{i}(z)+z^{-1} \Theta(z)$, where

$$
\Theta(z)=\left(G_{i, 1}(z)+G_{i, 2}(z)\right)\left(1-z^{-1}\right)^{-1}
$$

and hence

$$
E_{\max }=\lim _{i \rightarrow \infty}\left|E_{i}(z)\right|=\lim _{i \rightarrow \infty}\left|U_{i}(z)+z^{-1} \Theta(z)\right| .
$$

If $z^{-1} \Theta(z)$ and $U_{i}(z)$ have no phase difference when $i \rightarrow \infty$, then we have

$$
E_{\max }=\lim _{i \rightarrow \infty}\left|E_{i}(z)\right|=\lim _{i \rightarrow \infty}\left|U_{i}(z)\right|+\left|z^{-1} \Theta(z)\right|=2 u^{\infty}+\left|z^{-1} \Theta(z)\right| .
$$


Based on the steady state analysis, we have another approximation when $i \rightarrow \infty$, i.e.,

$$
\left\{\begin{aligned}
\left|H_{i-1}(z)\right| & \approx \frac{H_{\max }}{E_{\max }}\left|E_{i}(z)\right|=\frac{2 u^{\infty}}{\omega E_{\max }}\left|E_{i}(z)\right|, \\
\left|H_{i}(z)\right| & \approx \frac{H_{\max }}{E_{\max }}\left|E_{i}(z)\right|=\frac{2 u^{\infty}}{\omega E_{\max }}\left|E_{i}(z)\right| .
\end{aligned}\right.
$$

Now (14) is used for small $i$ 's while (24) is used for large $i$ 's. Combining (14) and (24) by choosing appropriate $\varphi_{j k}$ values yields a more accurate prediction (19).

\section{A.2 Proof for Lemma 2 and Corollary 1}

Proof. Without losing generality, we assume that the order quantity sequence is given in the form of

$$
u_{i}(t)=A_{i} \sin (\omega t)+B_{i} \cos (\omega t)+C,
$$

where $\left|U_{i}(z)\right|=\left(A_{i}^{2}+B_{i}^{2}\right)^{1 / 2}$ and $C=u^{\infty}$. Now suppose $\left|E_{i}(z)\right|>0,\left|E_{i}(z)\right|+e_{i}^{\infty}>0$. When $\left|E_{i}(z)\right| \leq e_{i}^{\infty}$, and then $e_{i}(t)$ will not be truncated by $N$, i.e., $N\left(e_{i}(t)\right)=e_{i}(t)$. Then the system falls into linear phase. We can directly obtain the amplification for the nonlinear module in the linear phase, i.e.,

$$
R_{i}^{N}=\left|\frac{H_{i}(z)}{\tilde{H}_{i-1}(z)}\right|=\left|z^{-1} \frac{\left(1-z^{-1}\right)^{-1}}{1+z^{-1}\left(1-z^{-1}\right)^{-1}}\right|=\left|z^{-1}\right|=1 .
$$

which proves (i).

Equation (25) implies that the nonlinear module will not change the amplitude in the linear phase. As for (ii), however, if $\left|E_{i}(z)\right|>e_{i}^{\infty}$, the system is switched into the nonlinear phase where the amplitudes would be affected. Now we have $N\left(e_{i}(t)\right)=\max \left\{e_{i}(t), 0\right\}=e(t) \cdot 1_{t \in\left[-t_{0}, \pi / \omega+t_{0}\right]}$. It follows from DFA and (13) that

$$
\begin{aligned}
C & =\frac{\omega}{2 \pi} \int_{-t_{0}}^{\frac{2 \pi}{\omega}-t_{0}} N\left(e_{i}(t)\right) \mathrm{d} t=\frac{e_{i}^{\infty}}{2}+\frac{e_{i}^{\infty}}{\pi} \omega t_{0}+\frac{\left|E_{i}(z)\right|}{\pi} \cos \left(\omega t_{0}\right), \\
\frac{A_{i}+j B_{i}}{\left|E_{i}(z)\right|} & =\frac{j \omega}{\pi\left|E_{i}(z)\right|} \int_{-t_{0}}^{\frac{2 \pi}{\omega}-t_{0}} N\left(e_{i}(t)\right) \mathrm{d} t \\
& =\frac{1}{2}+\frac{\omega t_{0}}{\pi}+\frac{2 e_{i}^{\infty}}{\pi\left|E_{i}(z)\right|} \cos \left(\omega t_{0}\right)-\frac{1}{2 \pi} \sin \left(2 \omega t_{0}\right) .
\end{aligned}
$$

It can be obtained from (27) that $B_{i}=0$ and thus $A_{i}=\left|U_{i}(z)\right|$. Combining (26) and (27) and substituting $\theta_{i}=\omega t_{i}$ gives the following result:

$$
\begin{aligned}
\left|U_{i}(z)\right| & =\left(\frac{1}{2}+\frac{\theta_{i}}{\pi}+\frac{\sin \left(2 \theta_{i}\right)}{2 \pi}\right)\left|E_{i}(z)\right|=g_{1}\left(\theta_{i}\right)\left|E_{i}(z)\right|, \\
C & =\left(\frac{1}{\pi}\left(\cos \theta_{i}+\theta_{i} \sin \theta_{i}\right)+\frac{1}{2} \sin \theta_{i}\right)\left|E_{i}(z)\right|=g_{2}\left(\theta_{i}\right)\left|E_{i}(z)\right|,
\end{aligned}
$$

Moreover, for cumulative orders, we derive in time domain and obtain that

$$
\begin{aligned}
\left|\tilde{H}_{i-1}(z)\right| & =\left(\left(\frac{\left|U_{i}(z)\right|}{2 \sin (\omega / 2)}\right)^{2}+\left|E_{i}(z)\right|\left(\left|E_{i}(z)\right|-\left|U_{i}(z)\right|\right)\right)^{1 / 2} \\
\left|H_{i}(z)\right| & =\left(\left(\frac{1}{2}\left|U_{i}(z)\right|\right)^{2}+\left(\frac{1}{2}\left|U_{i}(z)\right| \cot (\omega / 2)\right)^{2}\right)^{1 / 2}=\frac{\left|U_{i}(z)\right|}{2 \sin (\omega / 2)}
\end{aligned}
$$


Given $\left|\tilde{H}_{i-1}(z)\right|$, we can solve (28)-(31) to obtain $\left|H_{i}(z)\right|$ and the nonlinear gain

$$
R_{i}^{N}=\left|\frac{H_{i}(z)}{\tilde{H}_{i-1}(z)}\right|=\left(1+4\left|E_{i}(z)\right|\left(\left|E_{i}(z)\right|-\left|U_{i}(z)\right|\right)\left|U_{i}(z)\right|^{-2} \sin ^{2}(\omega / 2)\right)^{-1 / 2} .
$$

Since $\left|E_{i}(z)\right| \geq 0,\left|U_{i}(z)\right| \geq 0$, we need to show $\left|E_{i}(z)\right| \geq\left|U_{i}(z)\right|$. When $e_{i}(t)$ is not truncated by $N$, we have $\left|E_{i}(z)\right|=\left|U_{i}(z)\right|$. Otherwise, note that $\mathrm{d} g_{1}\left(\theta_{i}\right) / \mathrm{d} \theta_{i}=\left(1+\cos \left(2 \theta_{i}\right)\right) / \pi>0$ when $\theta_{i}=\omega t_{i} \in$ $(-\pi / 2, \pi / 2)$, which implies that $g_{1}\left(\theta_{i}\right)$ is monotonically increasing. Then since $g_{1}(\pi / 2)=1$, it follows that $g_{1}\left(\theta_{i}\right) \leq 1$ and the Lemma follows from (25) and (28).

Finally, combining (28), (29), (30) and (31) leads to Corollary 1.

\section{A.3 Proof for Proposition 4}

Proof. When $R_{i}^{L}>1$, suppose that $\left|U_{0}(z)\right|$ is sufficiently small such that $\left|E_{0}(z)\right|<e_{0}^{\infty 3}$. Then we are in the linear phase where $e_{i}(t)$ is not truncated by $N$. It follows from (25) that $R_{i}^{N}=1$ and so

$$
\left|\frac{H_{i}(z)}{H_{i-1}(z)}\right|=\left|\frac{\tilde{H}_{i-1}(z)}{H_{i-1}(z)}\right|\left|\frac{H_{i}(z)}{\tilde{H}_{i-1}(z)}\right|=R_{i}^{L} R_{i}^{N}=R_{i}^{L}>1 .
$$

In the linear phase, $\left|E_{i}(z)\right|=\left|U_{i}(z)\right|$, which implies that the amplitude $\left|U_{i}(z)\right|$ (corresponding to $\left|H_{i}(z)\right|$ ) will increase with respect of $i$ until $i=i_{0}$. Generally, $\theta_{i}$ and $R_{i}^{N}$ are invariant until $i>i_{0}$. To find the trend of $\theta_{i}$, first observe that $\left|\tilde{H}_{i-1}(z)\right|$ is a function of $\theta_{i}$, and from (28)-(30) it can be expressed in the following form:

$$
\left|\tilde{H}_{i-1}\left(z \mid \theta_{i}\right)\right|=\frac{C}{g_{2}\left(\theta_{i}\right)}\left(\left(\frac{g_{1}\left(\theta_{i}\right)}{2 \sin (\omega / 2)}\right)^{2}+\left(1-g_{1}\left(\theta_{i}\right)\right)\right)^{1 / 2} .
$$

Simple calculus shows that the derivative of (33) with respect to $\theta_{i}$ is negative when $\theta_{i} \in(-\pi / 2, \pi / 2)$, see Fig. 8a as an illustration. When $i>i_{0}$ but not too large, $R_{i}^{N}$ is still close to 1 such that $R_{i}^{L} R_{i}^{N}>1$, and hence $\left|U_{i}(z)\right|$ and $\left|\tilde{H}_{i-1}(z)\right|$ will still be increasing and $\theta_{i} \downarrow$. Note that $g_{2}\left(\theta_{i}\right)$ is monotonically increasing since $\mathrm{d} g_{2}\left(\theta_{i}\right) / \mathrm{d} \theta_{i}=\cos \left(\theta_{i}\right)\left(\theta_{i} / \pi+1 / 2\right)>0$ when $\theta_{i} \in(-\pi / 2, \pi / 2)$. Then (29) implies that $\left|E_{i}(z)\right| \uparrow$ as $\theta_{i} \downarrow$.

Now we discuss how $R_{i}^{N}$ will change as $i \uparrow$. Equations (28), (29) and (32) tell that $R_{i}^{N}$ is also a function of $\theta_{i}$, and it can be expressed as follows:

$$
R_{i}^{N}\left(\theta_{i}\right)=\left(1+4 \sin ^{2}(\omega / 2)\left(g_{1}^{-2}\left(\theta_{i}\right)-g_{1}^{-1}\left(\theta_{i}\right)\right)\right)^{-1 / 2} .
$$

It can be shown by simple calculus that (34) is monotonically increasing with respect to $\theta_{i}$ (see Fig. $8 \mathrm{~b}$ ), and hence $R_{i}^{N} \downarrow$ as $\theta_{i} \downarrow$. As a consequence, when $i>i_{0}$ and increases, $R_{i}^{N}$ will be less than 1 and decreasing until $R_{i}^{L} R_{i}^{N}=1$, after which $\left|H_{i}(z)\right|=\left|H_{i-1}(z)\right|$ and $\left|U_{i}(z)\right|=\left|U_{i-1}(z)\right|$. As a result, $\left|U_{i}(z)\right|$ will be invariant afterwards and bring the system into the asymptotic steady state.

In summary, when $i<i_{0}$, the amplitude of order fluctuations will grow exponentially with rate $R_{i}^{L}$; when $i \geq i_{0}$, the growth rate will gradually decrease to 1 , implying that the amplitude of order fluctuations will converge to a bounded value. On the other side, if $R_{i}^{L}=1$, since the first supplier lies in the linear phase, we always have $\left|H_{i}(z)\right| /\left|H_{i-1}(z)\right|=R_{i}^{L} R_{i}^{N}=1$ and thus the amplitudes will remain unchanged.

Moreover, (22) can be used for predicting the equilibrium value $U_{\max }$. Specifically, since $R_{i}^{L}$ 's are determined by system parameters $l_{i}, \omega$ and forecasting method, they can be calculated in advance. Then solving

\footnotetext{
${ }^{3}$ This is reasonable since customer demand usually has small fluctuations compared to upstream suppliers.
} 


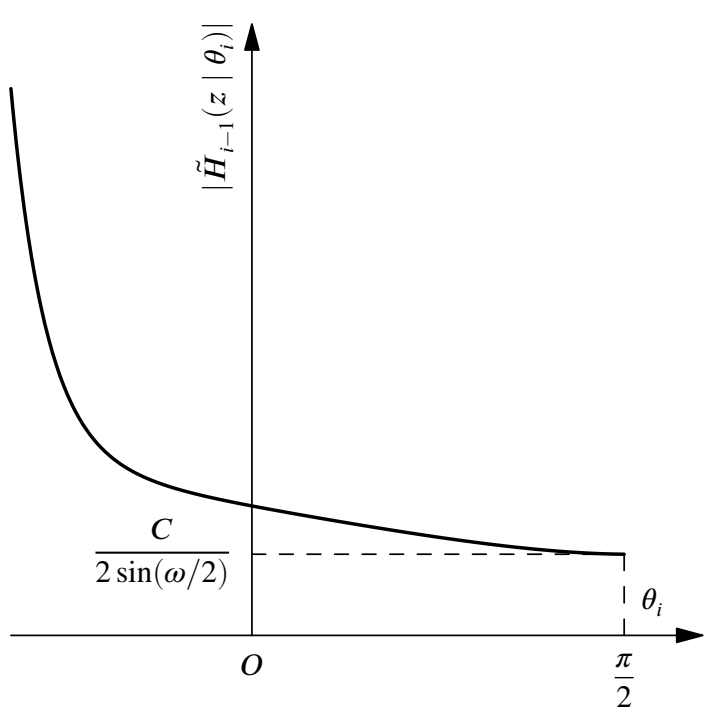

(a) $\left|\tilde{H}_{i-1}\left(z \mid \theta_{i}\right)\right|$

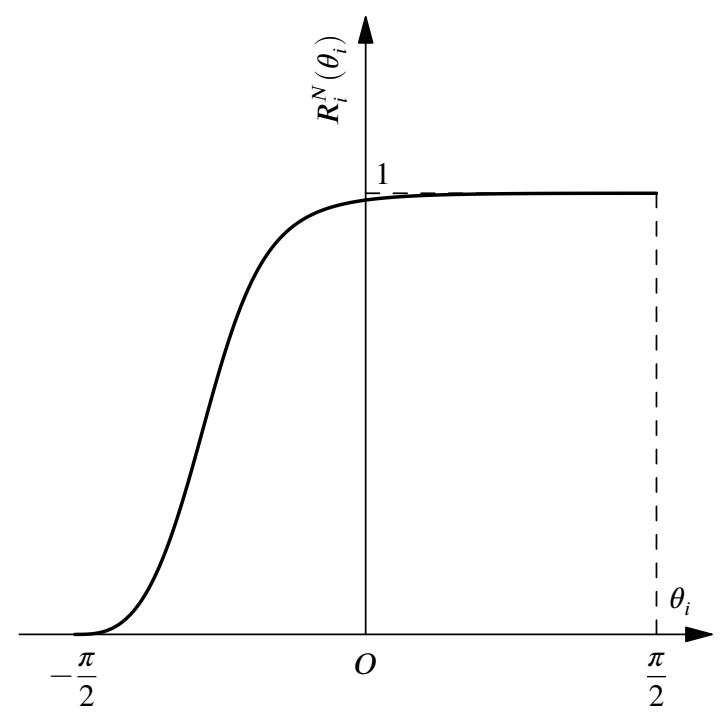

(b) $R_{i}^{N}\left(\theta_{i}\right)$

Figure 8: Monotonicity of the functions with respect of $\theta_{i} \in(-\pi / 2, \pi / 2) .\left|\tilde{H}_{i-1}\left(z \mid \theta_{i}\right)\right|$ is monotonically decreasing and tends to $+\infty$ and $C /(2 \sin (\omega / 2))$ when $\theta_{i} \downarrow-\pi / 2$ and $\uparrow \pi / 2$ respectively. $R_{i}^{N}\left(\theta_{i}\right)$ is monotonically increasing and tends to 0 and 1 when $\theta_{i} \downarrow-\pi / 2$ and $\uparrow \pi / 2$ respectively.

$R_{i}^{L} R_{i}^{N}\left(\theta_{i}\right)=1$ gives the corresponding $\theta_{i}$ in steady state. Observe that $U_{\max } R_{i}^{L}=\left|\tilde{H}_{i-1}\left(z \mid \theta_{i}\right)\right|, i \rightarrow \infty$, and thus $U_{\max }$ can be obtained from $\theta_{i}$. Note that such $U_{\max }$ is a lower bound of its exact value since it is obtained from quasi-linear approximation.

\section{A.4 Proof for Propostion 5}

Proof. Now, for order-up-to policy, we have that $P\left(z^{-1}\right)=-1, Q\left(z^{-1}\right)=0$. To obtain $s$, note that the nonlinear function $N\left(e_{i}(t)\right)=\max \left\{e_{i}(t), 0\right\}, i=1,2, \ldots$ only provides a simple truncation without any time shifting. Therefore, simply letting $\mathcal{N}\left(\bar{e}_{i}(t), u^{\infty}, \omega\right)=1$ yields a transfer function for the other linear components in the system including all the information about time shifting, i.e.,

$$
\left(\frac{U_{i}(z)}{U_{i-1}(z)}\right)_{\text {linear }}=\left(1+S\left(z^{-1}\right)\left(1-z^{-1}\right)\right) \cdot z^{-1} .
$$

Note that $\left(1+S\left(z^{-1}\right)\left(1-z^{-1}\right)\right)$ provides no time shift since its a constant plus the first difference of weighted summation and thus the time shift between $U_{i}(z)$ and $U_{i-1}(z)$ is reflected by $z^{-1}$, implying that $s=-1$. Now, substituting the above equation into (16) gives

$$
U_{i}(z)=\mathcal{N}\left(U_{i}(z)\left(1+S\left(z^{-1}\right)\right)\right) .
$$

We show that the sampling function is the only stable solution to (35). First we show in the time domain that sampling function $\mathbf{u}_{i}$ with period $2 \pi / \omega$ is a solution. For the ease of notation we suppose $2 \pi / \omega$ is an integer. Consider one time period $[0,2 \pi / \omega]$, without losing generality, and suppose that at some time $k=0$ we have $u_{i}(0)=a$, where $a$ is the magnitude of this impulse. Now assume the period $2 \pi / \omega$ is large enough 
such that the impacts of impulses in history are negligible. Then denote $\mathbf{v}_{i}:=\mathbf{u}_{i}(1+S(\Delta))$, and thus

$$
v_{i}(0)=a(1+S(0)), \quad v_{i}(k)=\frac{a}{k !} S^{(k)}(0), k \in[1,2 \pi / \omega],
$$

where the subscript $(k)$ indicates the $k$ th-order derivative.

Note that $(k !)^{-1} S^{(k)}(0)$ for any $k$ is simply the coefficient of historical order $u(t-k)$. When these coefficients are monotonically decreasing with the exponent degree in the polynomial $S$ (i.e., the more ancient order history has less impact on demand forecast) and all histories are non-negatively correlated with the forecast; i.e.,

$$
\frac{1}{k !} S_{i}^{(k)}(0) \geq \frac{1}{(k+1) !} S_{i}^{(k+1)}(0) \geq 0, \quad k=0,1,2, \ldots,
$$

then, condition (36) leads to

$$
\min _{k \in[1,2 \pi / \omega]}\left\{v_{i}(0)-v_{i}(k)\right\} \geq a,
$$

which means by an appropriate truncation, $\mathbf{u}_{i}=\bar{N}\left(\mathbf{v}_{i}\right)$ can be a sampling function with magnitude $a$. Moreover, Proposition 1 of Li and Ouyang (2011) implies that such a truncation of $\bar{N}$ always exists. Thus (35) always hold.

Next we show that any other possible solution are unstable, and they will eventually converge to a sampling function. It is sufficient to show a periodic sequence solution $\mathbf{u}_{i}^{\prime}$ with $u_{i}^{\prime}(0)=a$ and $u_{i}^{\prime}(1)=b$ is unstable within $[0,2 \pi / \omega]$. As analyzed above, to satisfy (35), the following equation must holds to ensure the same difference between $u_{i}^{\prime}(0)$ and $u_{i}^{\prime}(1)$ after truncation.

$$
a S(0)=a S^{\prime}(0)+b S(0) .
$$

By perturbing this solution by arbitrarily small coupled impulses $\delta \mathbf{u}_{i}^{\prime}$ with $\delta u_{i}^{\prime}(0)=\epsilon, \delta u_{i}^{\prime}(1)=-\epsilon$ and $\delta u_{i}^{\prime}(k)=0$ for all $k \in[2,2 \pi / \omega]$, the first two elements of $\left(\mathbf{v}_{i}^{\prime}+\delta \mathbf{v}_{i}^{\prime}\right)=\left(\mathbf{u}_{i}^{\prime}+\delta \mathbf{u}_{i}^{\prime}\right)(1+S(\Delta))$ are approximately

$$
\begin{aligned}
& v_{i}^{\prime}(0)+\delta v_{i}^{\prime}(0)=(a+\epsilon)(1+S(0)), \\
& v_{i}^{\prime}(1)+\delta v_{i}^{\prime}(1)=(b-\epsilon)(1+S(0))+(a+\epsilon) S^{\prime}(0) .
\end{aligned}
$$

To test the stability, it is sufficient to simply compare the difference between the first two elements before and after multiplying $(1+S(\Delta))$; i.e., $u_{i}^{\prime}(0)+\delta u_{i}^{\prime}(0)-\left(u_{i}^{\prime}(1)+\delta u_{i}^{\prime}(1)\right)$ and $v_{i}^{\prime}(0)+\delta v_{i}^{\prime}(0)-\left(v_{i}^{\prime}(1)+\delta v_{i}^{\prime}(1)\right)$. Since

$$
u_{i}^{\prime}(0)+\delta u_{i}^{\prime}(0)-\left(u_{i}^{\prime}(1)+\delta u_{i}^{\prime}(1)\right)=a-b+2 \epsilon
$$

and it follows from (36) that

$$
\begin{aligned}
v_{i}^{\prime}(0)+\delta v_{i}^{\prime}(0)-\left(v_{i}^{\prime}(1)+\delta v_{i}^{\prime}(1)\right) & =a-b+2 \epsilon+\epsilon S(0)+\epsilon\left(S(0)-S^{\prime}(0)\right) \\
& >a-b+2 \epsilon
\end{aligned}
$$

which implies that the perturbation is enlarged after the linear operation and thus $\mathbf{u}_{i}^{\prime}$ is an unstable solution. As a result, the difference between $u_{i}^{\prime}(0)$ and $u_{i}^{\prime}(1)$ will keep growing until one of them is 0 and finally the order sequence converges to a sampling function. This completes the proof. 


\section{References}

Adelson, R., 1966. The dynamic behaviour of linear forecasting and scheduling rules. Operational Research Quarterly 17 (4), 447-462.

Baganha, M. P., Cohen, M. A., 1998. The stabilizing effect of inventory in supply chains. Operations Research 46 (3), 572-583.

Blaquiere, A., 2012. Nonlinear system analysis. Elsevier.

Blinder, A. S., 1986. Can the production smoothing model of inventory behavior be saved? The Quarterly Journal of Economics 101 (3), 431-453.

Cachon, G. P., Randall, T., Schmidt, G. M., 2007. In search of the bullwhip effect. Manufacturing \& Service Operations Management 9 (4), 457-479.

Chen, F., Ryan, J. K., Simchi-Levi, D., 2000a. The impact of exponential smoothing forecasts on the bullwhip effect. Naval Research Logistics 47 (4), 269-286.

Chen, F., Ryan, J. K., Simchi-Levi, D., 2000b. Quantifying the bullwhip effect in a simple supply chain: the impact of forecasting, lead times, and information. Management science 46 (3), 436-443.

Clark, A. J., Scarf, H., 1960. Optimal policies for a multi-echelon inventory problem. Management science 6 (4), 475-490.

Córdoba, A., 1989. Dirac combs. letters in mathematical physics 17 (3), 191-196.

Daganzo, C. F., 2003. A theory of supply chains. Springer.

Daganzo, C. F., 2004. On the stability of supply chains. Operations Research 52 (6), 909-921.

Davis, H. T., 1962. Introduction to nonlinear differential and integral equations. Courier Dover Publications.

Dejonckheere, J., Disney, S. M., Lambrecht, M. R., Towill, D. R., 2003. Measuring and avoiding the bullwhip effect: a control theoretic approach. European Journal of Operational Research 147 (3), 567-590.

Dejonckheere, J., Disney, S. M., Lambrecht, M. R., Towill, D. R., 2004. The impact of information enrichment on the bullwhip effect in supply chains: a control engineering perspective. European Journal of Operational Research 153 (3), 727-750.

Deziel, D., Eilon, S., 1967. A linear production-inventory control rule. Production Engineer 46 (2), 93.

Edie, L. C., 1961. Car-following and steady-state theory for noncongested traffic. Operations Research 9 (1), 66-76.

Elmaghraby, S. E., 1966. The design of production systems. Reinhold Pub. Corp.

Forrester, J. W., 1958. Industrial dynamics: a major breakthrough for decision makers. Harvard business review 36 (4), $37-66$.

Fu, D., Aghezzaf, E.-H., Keyser, R. D., 2014. A model predictive control framework for centralized management of a supply chain dynamic system. Systems Science \& Control Engineering 2 (1), 250-260.

Gaur, V., Giloni, A., Seshadri, S., 2005. Information sharing in a supply chain under ARMA demand. Management Science 51 (6), 961-969.

Gilbert, K., 2005. An ARIMA supply chain model. Management Science 51 (2), 305-310.

Goodwin, J. S., Franklin, S. G., 1994. The beer distribution game: using simulation to teach systems thinking. Journal of Management Development 13 (8), 7-15.

Graves, S. C., 1999. A single-item inventory model for a nonstationary demand process. Manufacturing \& Service Operations Management 1 (1), 50-61.

Haykin, S., Van Veen, B., 2007. Signals and systems. John Wiley \& Sons.

Helbing, D., 2005. Production, supply, and traffic systems: a unified description. In: Traffic and Granular Flow '03. Springer Berlin Heidelberg, pp. 173-188.

Ivanov, D., Sokolov, B., 2013. Control and system-theoretic identification of the supply chain dynamics domain for planning, analysis and adaptation of performance under uncertainty. European Journal of Operational Research 224 (2), 313-323.

Jalaleddini, K., Aghdam, A. G., 2010. Elimination of limit cycles in wireless communication networks using three-level comparators. In: American Control Conference (ACC), 2010. IEEE, pp. 1847-1849.

Karlin, S., 1958. One stage inventory models with uncertainty. Studies in the mathematical theory of inventory and production, 109-134.

Kochenburger, R. J., 1950. A frequency response method for analyzing and synthesizing contactor servomechanisms. 
American Institute of Electrical Engineers, Transactions of the 69 (1), 270-284.

Lee, H. L., Padmanabhan, V., Whang, S., 1997. The bullwhip effect in supply chains. Sloan management review 38 (3), 93-102.

Lee, H. L., Padmanabhan, V., Whang, S., 2004. Information distortion in a supply chain: the bullwhip effect. Management science 50 (12_supplement), 1875-1886.

Li, Q., Disney, S. M., Gaalman, G., 2013. Avoiding the bullwhip effect using damped trend forecasting and the orderup-to replenishment policy. International Journal of Production Economics.

Li, X., Ouyang, Y., 2011. Characterization of traffic oscillation propagation under nonlinear car-following laws. Procedia-Social and Behavioral Sciences 17, 663-682.

Li, X., Peng, F., Ouyang, Y., 2010. Measurement and estimation of traffic oscillation properties. Transportation Research Part B: Methodological 44 (1), 1-14.

Li, X., Wang, X., Ouyang, Y., 2012. Prediction and field validation of traffic oscillation propagation under nonlinear car-following laws. Transportation research part B: methodological 46 (3), 409-423.

Ouyang, Y., 2007. The effect of information sharing on supply chain stability and the bullwhip effect. European Journal of Operational Research 182 (3), 1107-1121.

Ouyang, Y., 2014. Experimental study on using advance demand information to mitigate the bullwhip effect via decentralised negotiations. Transportmetrica B: Transport Dynamics (ahead-of-print), 1-19.

Ouyang, Y., Daganzo, C., 2006a. Characterization of the bullwhip effect in linear, time-invariant supply chains: Some formulae and tests. Management science 52 (10), 1544-1556.

Ouyang, Y., Daganzo, C., 2006b. Counteracting the bullwhip effect with decentralized negotiations and advance demand information. Physica A: Statistical Mechanics and its Applications 363 (1), 14-23.

Ouyang, Y., Daganzo, C., 2008. Robust tests for the bullwhip effect in supply chains with stochastic dynamics. European Journal of Operational Research 185 (1), 340-353.

Ouyang, Y., Lago, A., Daganzo, C. F., 2006. Taming the bullwhip effect: from traffic to supply chains. In: Torres, O. A. C., Morán, F. A. V. (Eds.), Bullwhip Effect in Supply Chains: A Review of Methods, Components, and Cases, Palgrave-MacMilan Series.

Ouyang, Y., Li, X., 2010. The bullwhip effect in supply chain networks. European Journal of Operational Research 201 (3), 799-810.

Vander Velde, W. E., 1968. Multiple-input describing functions and nonlinear system design. New York: McGraw-Hill. Vassian, H. J., 1955. Application of discrete variable servo theory to inventory control. Journal of the Operations Research Society of America 3 (3), 272-282.

Zhang, X., 2004. Evolution of ARMA demand in supply chains. Manufacturing \& Service Operations Management $6(2), 195-198$. 